\title{
Diffusion tensor imaging in differentiation metastatic from reactive axillary lymph nodes in patients with breast cancer
}

\author{
Ahmed Abdel Razek ${ }^{1}$, Waleed Elnahas ${ }^{1}$, and Mahmoud Abd El-Latif ${ }^{1}$ \\ ${ }^{1}$ Mansoura University Faculty of Medicine
}

February 9, 2021

\begin{abstract}
Purpose: To determine if diffusion tensor imaging (DTI) parameters can differentiate metastatic from reactive axillary lymph nodes (ALNs) in patients with breast cancer. Methods: Prospective study was done on 48 patients with breast cancer that were examined by DTI of the breast. The mean diffusivity (MD) and fractional anisotropy (FA) of ALNs were calculated by 2 observers. The final diagnosis was obtained by biopsy. Results: Metastatic nodes $(\mathrm{n}=50)$ had significantly lower MD ( $\mathrm{p}=$ $0.001,0.001)$ and higher FA $(\mathrm{p}=0.002,0.01)$ than reactive $(\mathrm{n}=20)$ nodes for both observers respectively. When threshed of MD $(0.875,0.815$ X10-3 mm2/s) was used for differentiation of both entities revealed accuracy $(80 \%, 81.4 \%)$ and had AUC of 0.920 and 0.918 by both observers respectively. When threshold FA $(0.565,0.645)$ was used for differentiation revealed an accuracy of $87.1 \%, 81.4 \%$ and had AUC of 0.860 and 0.870 by both observers respectively. Combined FA and MD had AUC $0.940,0.950$ ) with an accuracy of $91.4 \%, 92.9 \%$ for both observers respectively. Conclusion: Combined analysis DTI parameters FA may play a role in differentiation of metastatic from reactive ALNs in patients with breast cancer.
\end{abstract}

\section{Hosted file}

2-Mans-DTI-Axil LN8-EJR4-Med.rtf available at https://authorea.com/users/393481/articles/ 508075-diffusion-tensor-imaging-in-differentiation-metastatic-from-reactive-axillarylymph-nodes-in-patients-with-breast-cancer

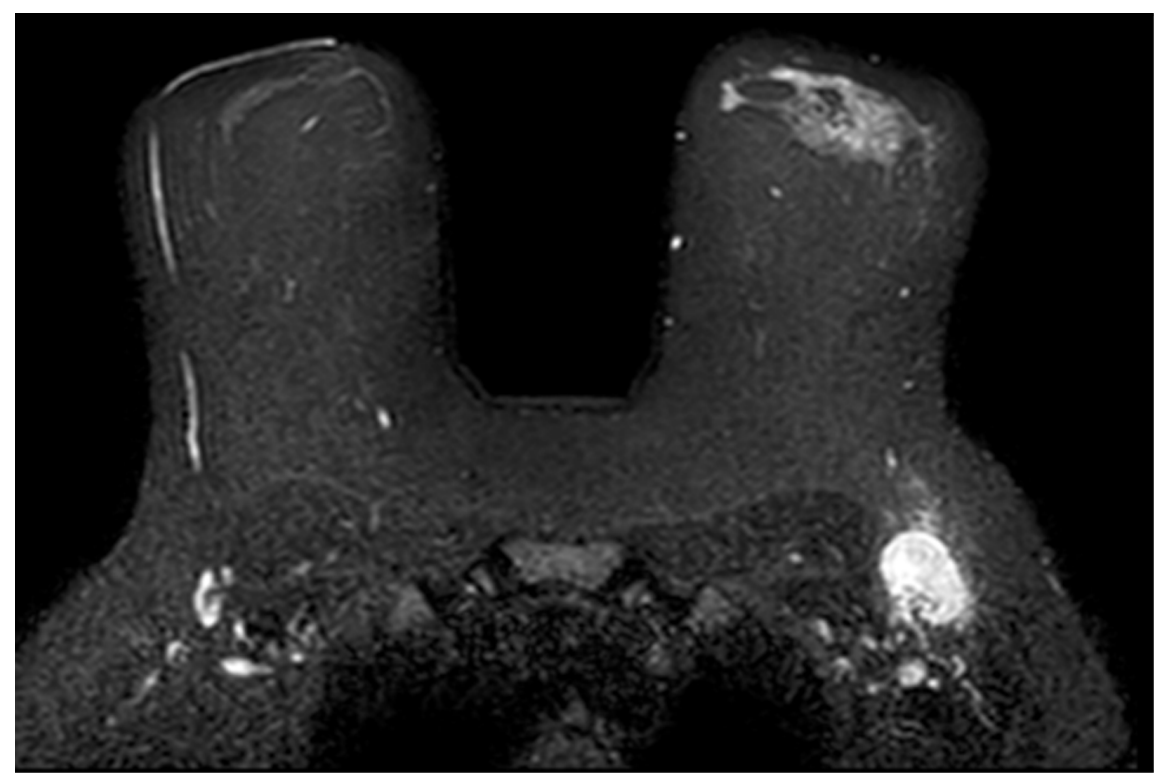



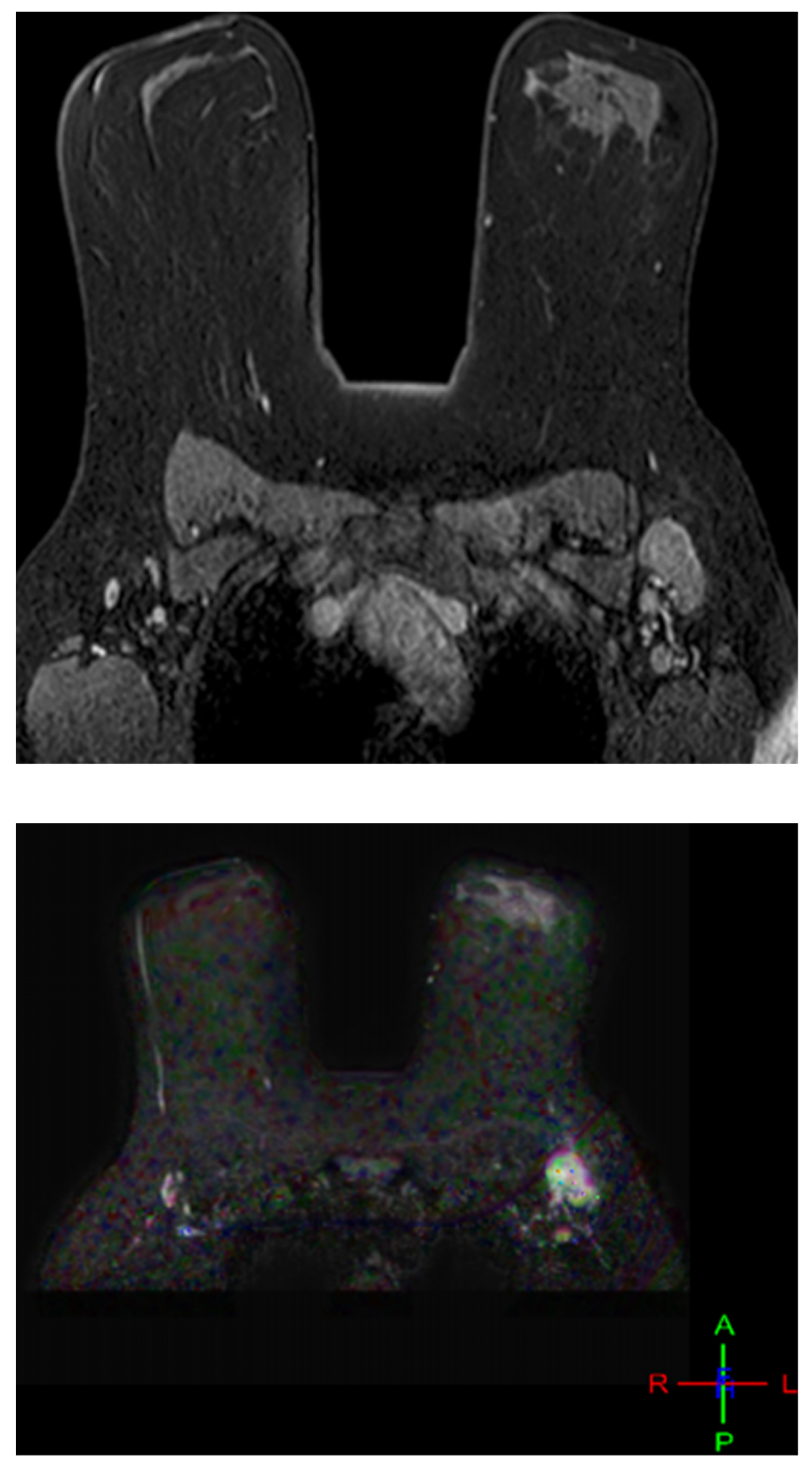


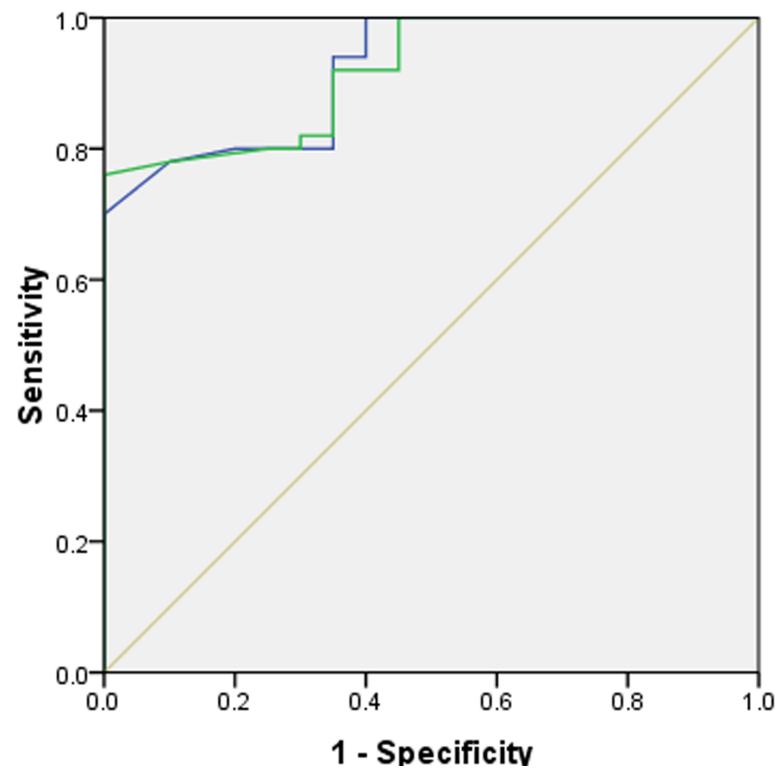

Source of the Curve - MD (First observer)

MD(second observer) Reference Line

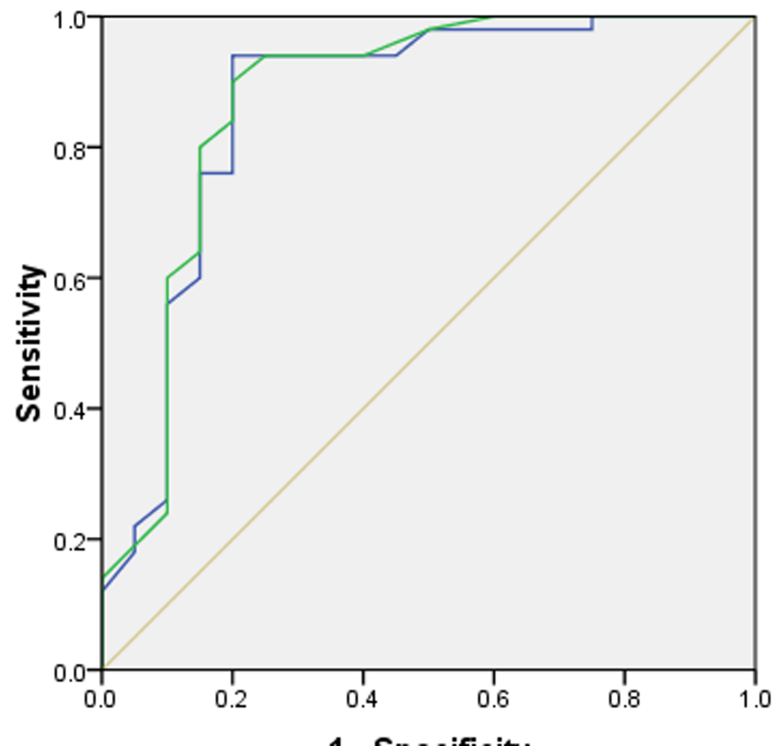

Source of the Curve

-FA (First observer)

FA (second observer)

Reference Line 

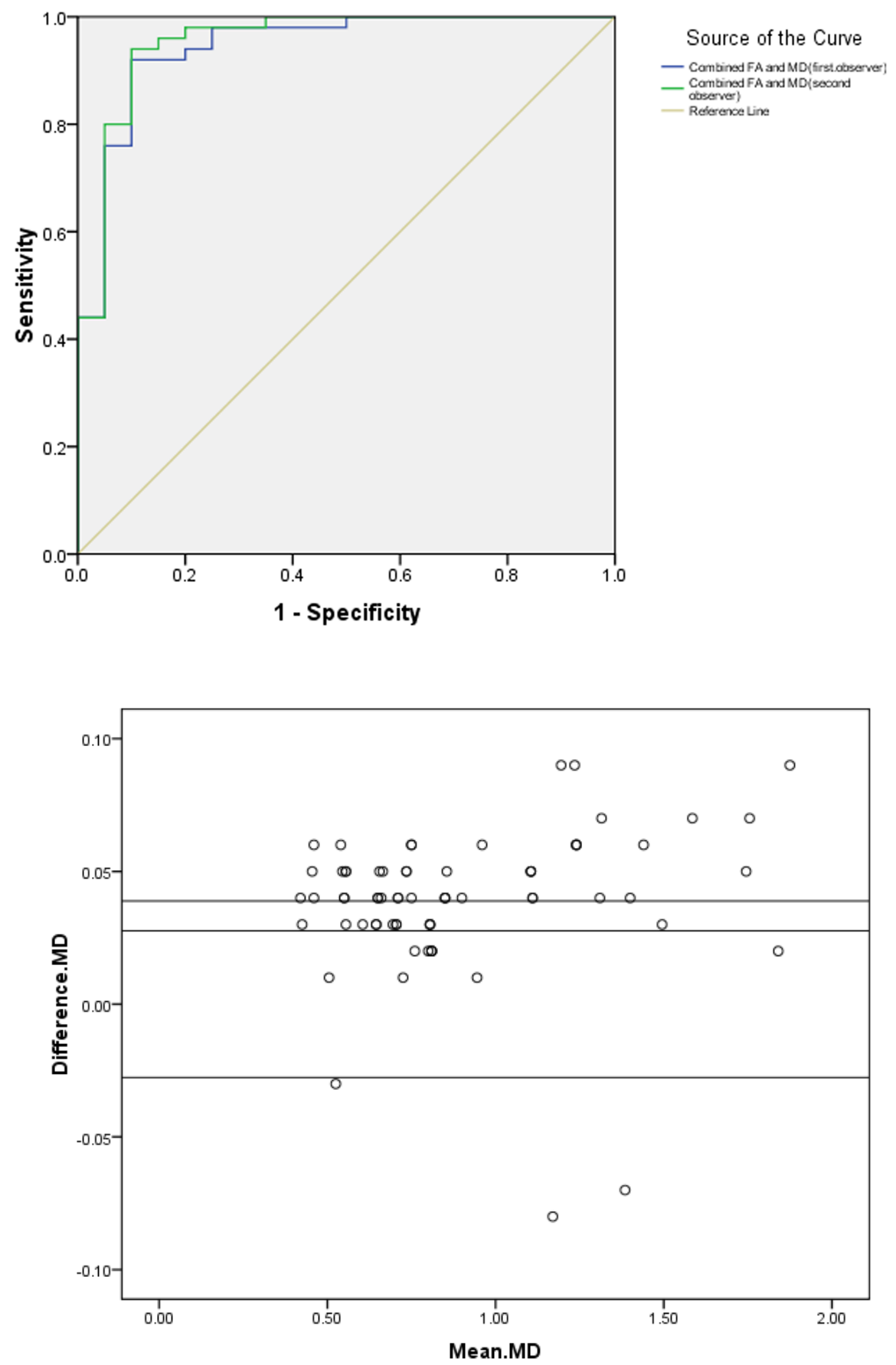


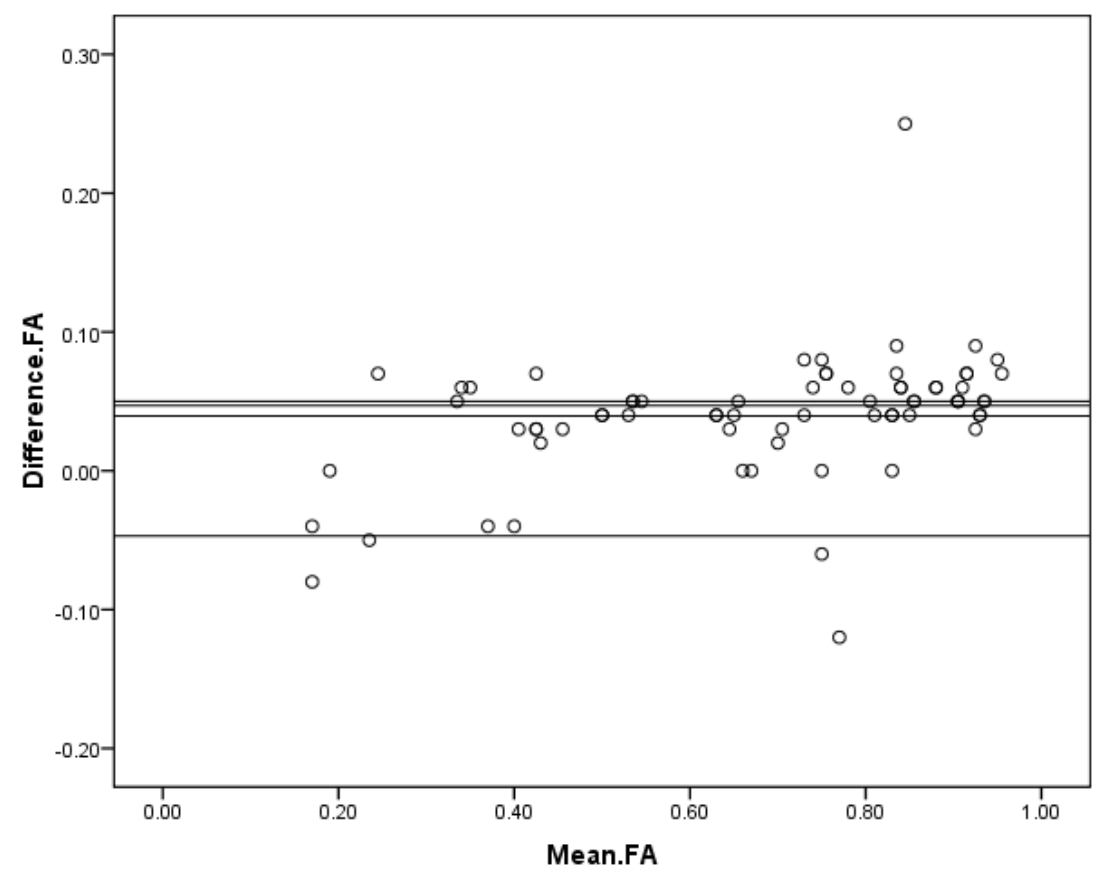

